\title{
Robotic magnetic navigation for ablation of human arrhythmias
}

\author{
This article was published in the following Dove Press journal: \\ Medical Devices: Evidence and Research \\ 19 September 2016 \\ Number of times this article has been viewed
}

\author{
Antoine $\mathrm{Da}$ Costa \\ Jean Baptiste Guichard \\ Cécile Roméyer-Bouchard \\ Antoine Gerbay \\ Karl Isaaz \\ North Hospital, Cardiology \\ Department University of Saint \\ Etienne Jean Monnet, Saint Etienne \\ Cedex 2, France
}

\begin{abstract}
Radiofrequency treatment represents the first choice of treatment for arrhythmias, in particular complex arrhythmias and especially atrial fibrillation, due to the greater benefit/ risk ratio compared to antiarrhythmic drugs. However, complex arrhythmias such as atrial fibrillation require long procedures with additional risks such as X-ray exposure or serious complications such as tamponade. Given this context, the treatment of arrhythmias using robotic magnetic navigation entails a technique well suited to complex arrhythmias on account of its efficacy, reliability, significant reduction in X-ray exposure for both patient and operator, as well as a very low risk of perforation. As ongoing developments will likely improve results and procedure times, this technology will become one of the most modern technologies for treating arrhythmias. Based on the literature, this review summarizes the advantages and limitations of robotic magnetic navigation for ablation of human arrhythmias.
\end{abstract}

Keywords: atrial fibrillation, ablation, arrhythmias, robotic magnetic navigation, stereotaxis

\section{Introduction}

Radiofrequency ablation (RFA) has become the treatment of choice for simple heart rhythm disorders because of its improved risk/benefit ratio compared to antiarrhythmics. ${ }^{1}$ Significant and decisive advances have been made over recent years in the treatment of complex arrhythmias, particularly in the management of atrial fibrillation (AFib). ${ }^{1-5}$ The treatment of these complex arrhythmias is carried out in expert centers that treat high patient volumes because manipulating the catheter in difficult clinical settings may lead to long and tedious, or even risky, procedures..$^{2-5}$ One of the main drawbacks of the manual approach is the technology of the catheters, whose movement is restricted by torque transmission, which in turn depends on the tortuosity of the vessels, the orientation of the catheter in the heart, and the rigidity or instability of the catheter. Moreover, electrophysiologists have seen their work change over time, the extension of indications having led to an increase in the number of procedures performed per operator. Operators will not only be more exposed to radiation but will also be unusually fatigued, and this may result in a loss of concentration. Such an inability to focus may delay analysis and subsequently prolong the procedure, and even increase the risk of complications. The treatment of AFib currently accounts for much of the work done in electrophysiology departments because of its prevalence $(30 \%-50 \%$ of procedures) and the poor efficacy of antiarrhythmic agents in this indication. ${ }^{5-8}$ The trend now favors robotic technologies, which are as effective as, or more effective than, manual RFA while offering better safety for both the patient and operator. Two
Correspondence: Antoine Da Costa North Hospital; Cardiology Department University of Saint Etienne Jean Monnet, FRANCE 42055 Saint

Etienne Cedex 2, France

Tel $+334778281 \quad 13$

Fax +33477828164

Email dakosta@orange.fr 
systems have been developed: the Robotic Magnetic Navigation System (RMNS) and the Hansen Sensei Robotic System (HSRS). ${ }^{9-26}$ This article sought to review robotic magnetic technology (stereotaxis) in the management and RFA of complex heart rhythm disorders in the light of the literature and our experience in the area.

\section{Description of RMNS (EPOCH)}

$\mathrm{EPOCH}$ is a technological platform that uses an orientable magnetic field to remotely guide a flexible catheter inside the heart. ${ }^{9-11}$ The orientable magnetic field comprises two giant 1.8ton magnets that are controlled from a computerized platform. Placed on either side of the fluoroscopy bed (Figure 1), these two magnets generate a magnetic field of $0.08-0.1$ tesla (depending on the initial choice) so that three small magnets built into the catheter's tip enable three-dimensional (3D) navigation (Figure 2 ). The magnetic field is applied to a theoretical heart volume of $20 \mathrm{~cm}$ by $20 \mathrm{~cm}$. The catheter's tip can be very accurately guided by means of a computerized vectorization system called "navigant" (Figure 3). This system works by aligning the catheter with the magnetic field generated. The catheter is then moved by changing the two magnets' orientation relative to each other. Advancing and retracting the catheter is piloted by a computerized motor drive (Cardiodrive; Stereotaxis Inc.) (Figure 4), while spatial orientation requires a computerized workstation (Navigant 2.1; Stereotaxis Inc.) (Figures 3 and 4).

Catheter advancement and retraction can be performed at any time and can be set between 1 and $9 \mathrm{~mm}$ using the arrows of a keyboard or a joystick. The third generation of EPOCH can tilt the magnets to orientations between $40^{\circ}$ in the left anterior oblique view and $30^{\circ}$ in the right anterior oblique view. By constantly applying the magnetic field during ablation, the tip of the catheter is kept in constant

A

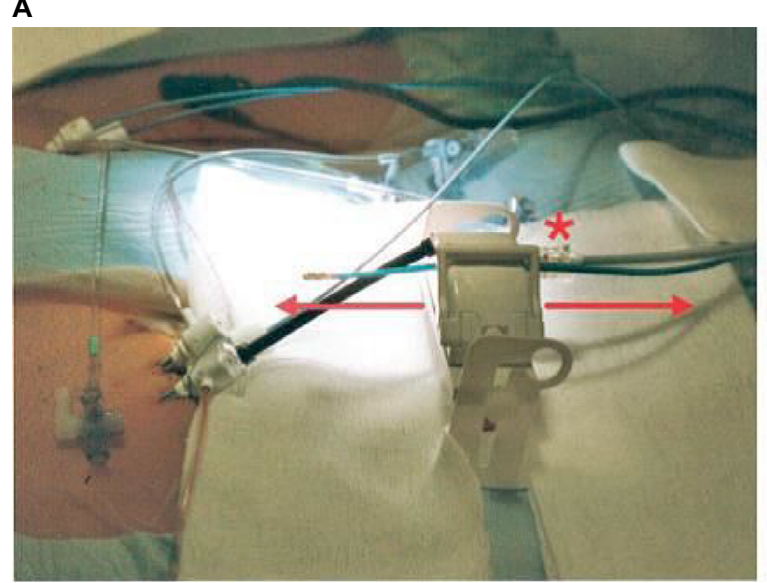

contact with the endocardial tissue throughout the cardiac cycle, thus improving the delivery of radiofrequency current. The flexible nature and low force (15-25 g)

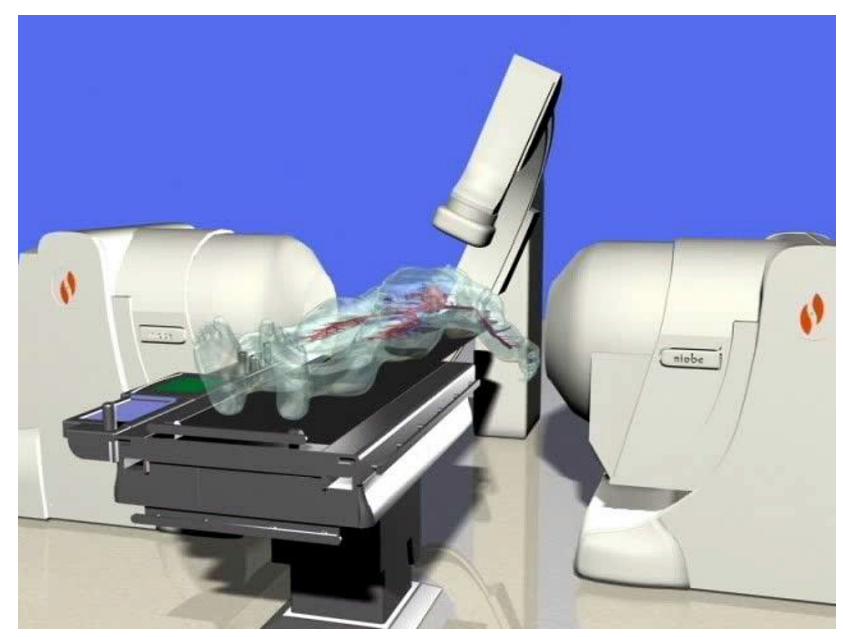

Figure I Simulation of the examination bed when the magnets are in active position and the patient is present.

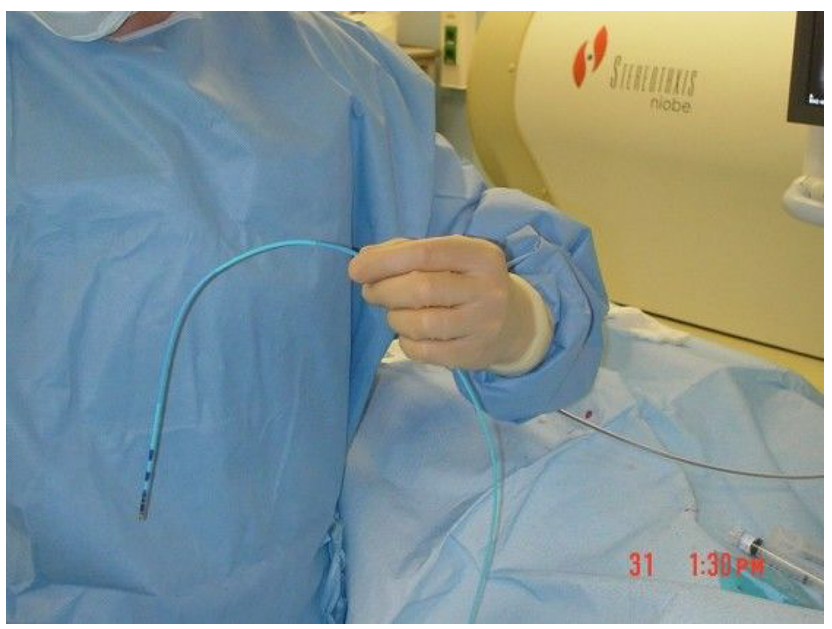

Figure 2 Irrigated magnetic catheter with three magnets built into the catheter's tip. Note: Note the extreme flexibility of the magnetic catheter.

\section{B}

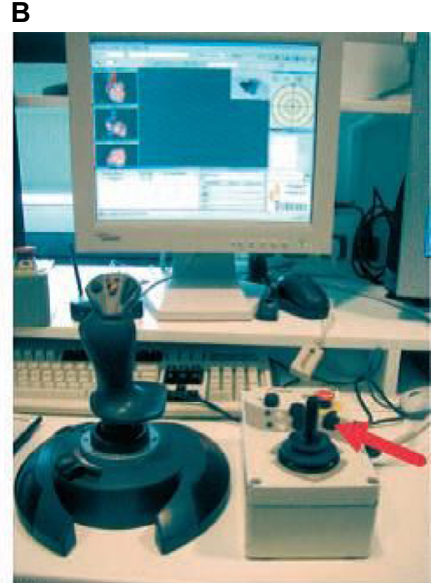

Figure 3 "QuickCAS" Cardiodrive system positioned in the patient's groin making it possible to advance the catheter by means of either the keyboard or, as above, a joystick (single arrow).

Notes: Advancing and retracting the catheter is piloted by a computerized motor drive (arrows); the asterisk corresponds to the cardiodrive motor connection (A); advancing and retracting the catheter is piloted by a joystick (B). 


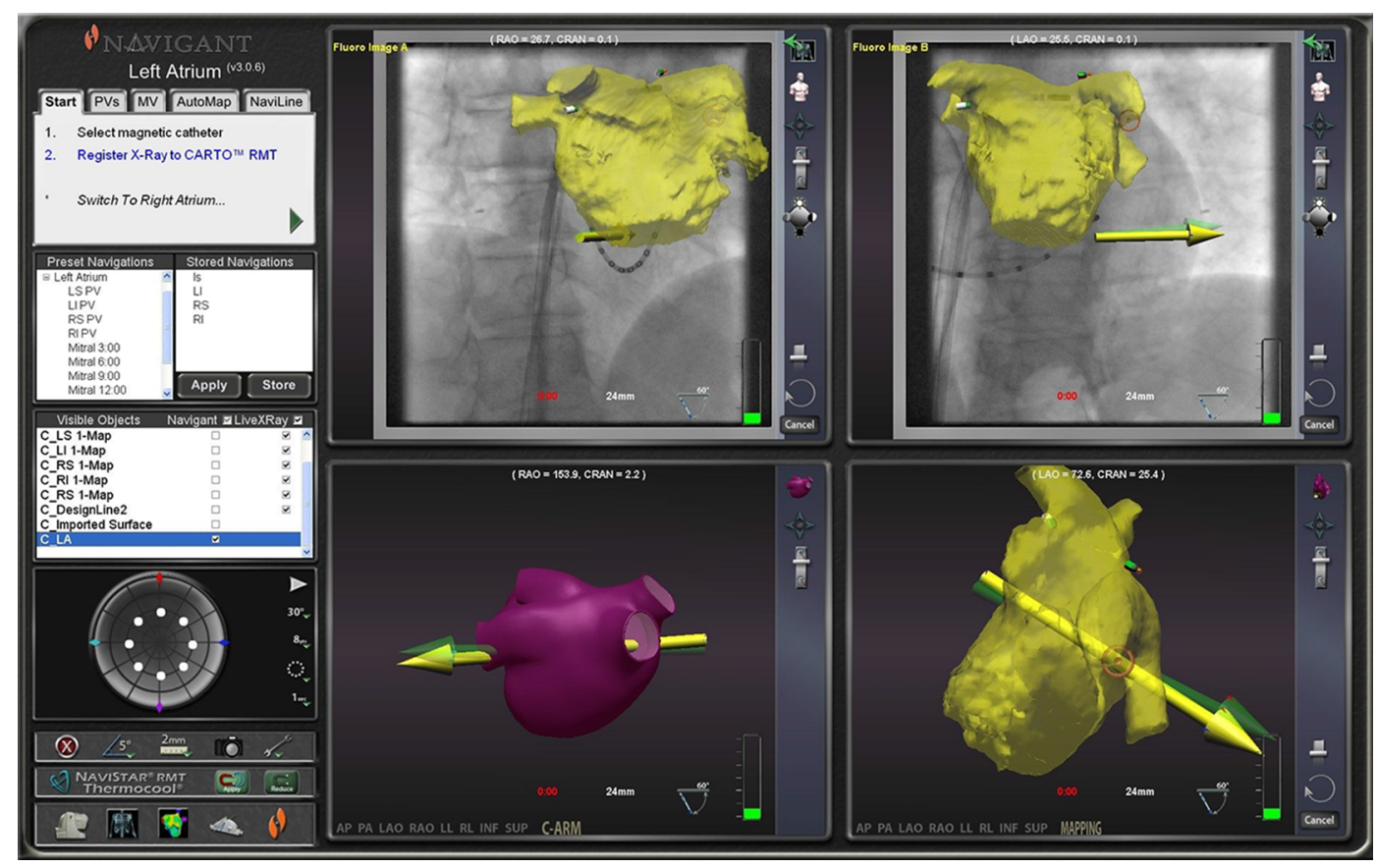

Figure 4 Navigant system screen that enables real-time navigation through different parts of the heart by simply orienting vectors from the keyboard where the doctor is positioned to work remotely from the patient.

Notes: The virtual vector is yellow. It is followed by the magnetic catheter in this example.
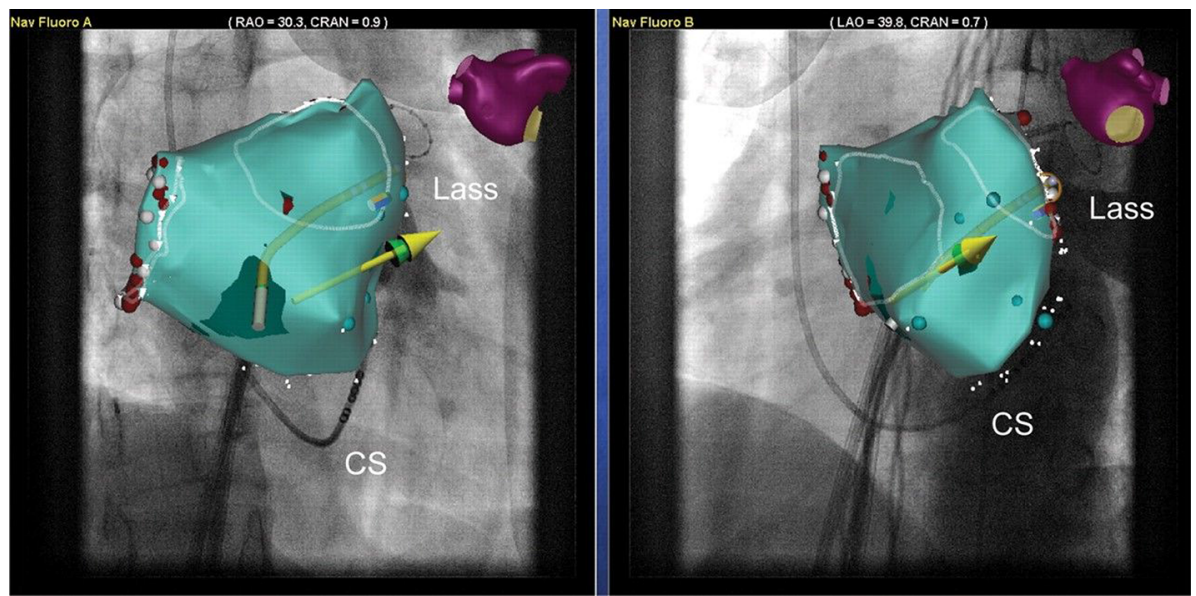

Figure 5 Navigant system screen that enables real-time navigation through different parts of the heart by simply orienting vectors from the keyboard where the doctor is positioned to work remotely from the patient.

Notes: The virtual vector is green. It is followed by the catheter vector in yellow. The lasso is shown (Lass). The green arrow corresponds to the virtual vector that it is followed by the magnetic catheter corresponding to the yellow arrow in this example.

Abbreviation: CS, coronary sinus catheter.

exerted by the magnetic field make navigation within the heart very safe, with the risk of perforation being almost zero. ${ }^{9}$ The system can also memorize certain orientations, such as the position of veins, and reuse these vectors during the examination in order to facilitate navigation and improve procedure times.

Automatic navigation is also possible using the "NaviLine" system, which enables automatic processing, for instance by automatically creating lines or circumferentially enclosing veins. The main step forward over recent months has been integrating a platform with Biosense Webster's 3D mapping systems CARTO XP, CARTOMERGE, and CARTO 3 System (Biosense Webster, Diamond Bar, CA, USA) (Figures 5 and 6), while the development of an irrigated $3.5 \mathrm{~mm}$ magnetic catheter has made it possible to employ this technology in the treatment of complex arrhythmias in the left atrium. The advantage of Carto 3 in this platform is visualizing all the catheters and being able to rapidly reconstruct anatomic structures using Lasso ${ }^{\circledR}$ Nav (Biosense Webster, Diamond Bar, CA, USA). 


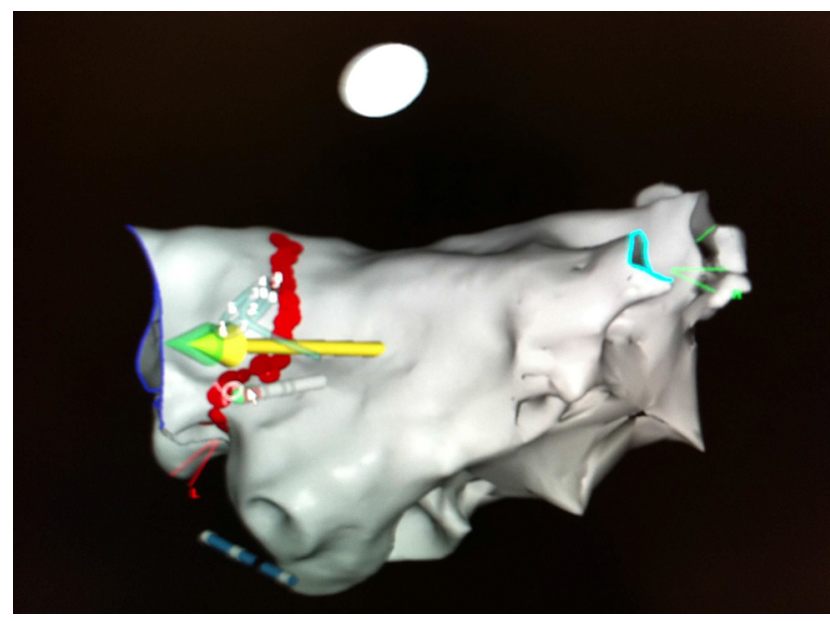

Figure 6 Integration of the Carto 3 RMN system with the Navigant system, which enables instantaneous navigation along with visualization of both the Navistar RMN catheter and the lasso.

Notes: The green arrow corresponds to the virtual vector that it is followed by the magnetic catheter corresponding to the yellow arrow.

Abbreviation: RMN, Robotic Magnetic Navigation.

\section{Experimental trials}

Tillander et $\mathrm{al}^{27}$ followed by Ram et $\mathrm{al}^{28}$ were the first to test the manipulation of catheters using magnetic fields, but their initial efforts were hampered by the low force of the magnetic field, size of the catheters, and lack of accurate 3D control. ${ }^{27-29}$ Later developments incorporated stereotactic localization and vector control by means of dedicated software. ${ }^{27}$

In 2002 , Faddis et $\mathrm{al}^{30}$ validated feasibility in an animal model. ${ }^{30,31}$ Several factors were investigated, including navigation, the force of catheter deflection, interference with the electrocardiogram signals analyzed during the procedure, and the efficacy of RFA. ${ }^{30,31}$ The authors demonstrated that the maximum force exerted on the catheter was $26.8 \mathrm{~g}$ compared to $31.4 \mathrm{~g}$ when using the manual method (ranging from 19.7 to $45.4 \mathrm{~g}$ ), while navigation was $90 \%$ accurate with regard to the targets tested. The magnetic catheter was then modified to improve the shape of its two segments - the flexible part and the more rigid part. This led to a precision of \pm 0.73 $\mathrm{mm}$. Navigation and the achievement of effective lesions in the pulmonary veins were tested in five animals including 30 veins. It was possible to navigate and obtain effective lesions in nearly $100 \%$ of cases. Interference with signals as studied using the signal-to-noise ratio was very low despite the distortion exerted on the catheter. More importantly, interference was nonsignificant compared with the manual method. That study had certain limitations, such as not being randomized and not analyzing the microscopic lesions generated by RFA. ${ }^{30}$

Faddis et $\mathrm{al}^{31}$ then conducted a clinical trial of feasibility and safety involving 31 patients requiring ablation for complex arrhythmia, three of whom had AFib. ${ }^{31}$ In that study, there was no difference in the quality of the signals when analyzed under blinded conditions against the manual method. ${ }^{31}$ Navigation in the heart cavities was possible in $213 / 215$ of the sites tested on the right (99\%) and 13/13 $(100 \%)$ of the sites tested on the left. The stimulation thresholds did not significantly differ between the manual method and RMNS. ${ }^{31}$ No complications were observed in the study, and ablation of the complex arrhythmias was possible in the seven patients tested ( $100 \%$ of cases). ${ }^{14}$ The advantages of the technique were underlined by the authors, who highlighted that there was no risk of perforation, the catheter had excellent stability, and it was possible to navigate in complex anatomies, as has been described in patients with congenital heart defects. ${ }^{30,31}$

\section{Clinical trials with RMNS (stereotaxis)}

Over the past 10 years, AFib ablation has become the predominant indication in centers of excellence, possibly representing up to $60 \%$ of interventions. The main difficulties during these procedures are the duration, which may exceed 4 hours, and the length of time that both the operator and patient are exposed to radiation. The advantages of the technique were underlined by different authors, who highlighted that there was no risk of perforation, the catheter had excellent stability, and it was possible to navigate in complex anatomies, as has been described in patients with congenital heart defects. ${ }^{30-33}$ Complications, and in particular severe complications, constitute one major limitation of these techniques, as was shown by Cappato et $\mathrm{al}^{34}$ in a registry of AFib ablation procedures conducted in 521 centers across 24 countries. ${ }^{34}$ Although AFib ablation was noticeably effective in $80 \%$ of cases, with a mean of 1.3-1.7 procedures per patient, the rate of major complications reported was $4.5 \%$ of cases, including $1 \%$ vascular accidents and $1.3 \%$ tamponade. ${ }^{34}$ The number of procedures to be conducted by the operator also needs to be taken into account because of the long-term risk of exposure to X-rays.

Several clinical trials have now been undertaken to evaluate RMNS in AFib. ${ }^{9,10,14-16}$ In the initial phase, Pappone et $\mathrm{al}^{9}$ evaluated the feasibility of RMNS in 40 AFib patients who required treatment by RFA. The authors demonstrated the feasibility and very good efficacy of the robotic technique. Application time was significantly reduced compared with the control group, although the trial was a case-control study. ${ }^{9}$ The operator highlighted the extreme stability of the magnetic catheter, which was especially useful for approaching the right veins. ${ }^{9}$ In the study, success was achieved in 38 of the 40 patients tested (95\% of cases). Di Biase et al ${ }^{10}$ reported 
contradictory results in a nonrandomized study involving 45 patients who were tested using RMNS with a nonirrigated $4 \mathrm{~mm}$ catheter. ${ }^{10}$ Although navigation was possible in most cases, the rate of successful vein disconnection was low ( $<90 \%$ of cases), mainly owing to the use of a nonirrigated catheter. ${ }^{10}$ The irrigated magnetic catheter has been marketed and used since March 2008. It enables an ablation technique that is comparable to the conventional method. ${ }^{14,16} \mathrm{Schmidt}$ et $\mathrm{al}^{20}$ also showed that the concept worked in AFib patients, highlighting the quality of navigation and stability of the catheter. These authors noted the problem of the learning curve, which makes initial procedures longer than the conventional method.

We too observed the same issue. The learning curve has to be overcome, and then significant benefits can be drawn from the technology. The first 100 patients we treated in our center in 2010 allowed us to gain a consistent and reproducible understanding of AFib ablation. Today, almost all our AFib RFA procedures are conducted by RMNS. There has not been a switch back to the manual method since the middle of 2010. To our knowledge, no randomized trials have compared the manual method against stereotaxis in the RFA of Afib.

A case-control comparative study was published by Lüthje et $\mathrm{al}^{14}$ involving 107 patients treated with RMNS compared against a group of 54 patients treated manually. Most veins were electrically disconnected in the two groups (in $90 \%$ of patients). The long-term success rate was similar between the two procedures: $66 \%$ with no AFib in the RMNS group versus $62.1 \%$ in the control group after a single procedure, while the success rate was $83 \%$ after $1.5 \pm 0.6$ procedures. ${ }^{14}$ Although the X-ray exposure rate was significantly lower in the robotic group, the procedure times were longer and so were the radiofrequency application times. ${ }^{14}$ The procedures in that study were conducted with the NIOBE II system, which has since been replaced by the much more accurate and faster EPOCH system. Miyazaki et $\mathrm{al}^{15}$ reported a case-control study involving 30 consecutive patients compared against 44 consecutive patients treated manually. The results were similar, but the RFA procedure times were longer in the RMNS group and the X-ray exposure times were $63 \pm 18$ minutes in the robotic group versus $47 \pm 17$ minutes $(P=0.0016)$ in the manual group. These contradictory results, particularly regarding X-ray exposure, were partly due to the fact that the operators had considerable experience with the manual technique, that no mapping system was used apart from a lasso-guided technique only, and, lastly, that the study was nonrandomized and conducted by less-experienced personnels. ${ }^{15}$ Pappone et $\mathrm{al}^{9}$ reported their experience involving 130 consecutive patients using an irrigated magnetic catheter (Thermocool Navistar-RMT; Biosense Webster, Diamond Bar, CA, USA). They showed that ablation was possible in 118 of 130 patients (practicability of $91 \%$ ) while only resorting to the manual catheter in 12 cases $(9 \%){ }^{25}$ Mean procedure time was $94.6 \pm 15.3$ minutes and the long-term success rate (15.3 \pm 5 months) was $81.4 \%$ in patients with paroxysmal AFib as against $67.3 \%$ in patients with permanent AFib. ${ }^{25}$ No major complications were mentioned aside from two femoral arteriovenous fistulas and one significant hematoma in the groin. ${ }^{16}$ Thus, automation of the treatment of complex arrhythmias by means of RFA is one of the most useful applications because of the reduced risk of both X-ray exposure and potential complications. The technique may nevertheless be applied to other arrhythmias. ${ }^{35}$ A literature review has been published with 41 references already validating the technique in all forms of arrhythmia, whether simple or complex. The results of these studies were published in the form of a meta-analysis. ${ }^{35}$ It emerged that the effectiveness rate in all ventricular or supraventricular tachycardias was the same as with the conventional method. The technique's advantages included, of course, a reduction in X-ray exposure, both for the operator and patient. ${ }^{35}$ Through this literature review, the authors thus highlighted that this new technology is feasible and accompanied by low risk, particularly for AFib ablation.

Although no randomized studies have been undertaken in the AFib treatment, a recent comparative study matching patients with the same characteristics has given a clear picture of the robotic technique's superiority. ${ }^{36}$ In that observational registry, Adragao et al ${ }^{36}$ compared RMNS against the manual method with regard to efficacy (arrhythmias recurrences $18.4 \%$ per year versus $22.3 \%$ per year; $P=0.108$ ), major complications $(0.7 \%$ versus $2.1 \% ; P=0.286)$, fluoroscopy time (12.9 \pm 0 versus $21 \pm 10$ minutes; $P<0.001$ ), and procedure time ( $213 \pm 58$ versus $152 \pm 52$ minutes; $P<0.001$ ), respectively, over a follow-up period of $2.6 \pm 1.5$ years. ${ }^{36}$ The technique's utility was thus demonstrated in terms of X-ray exposure, with similar efficacy and, it would seem, fewer serious complications. ${ }^{36}$ The evidence continues to build up in the AFib treatment. ${ }^{37}$ Jin et $\mathrm{al}^{37}$ have reported a prospective series of 726 patients, or 1006 procedures, of whom 240 patients underwent ablation more than once. Mean procedure times fell dramatically to $134 \pm 35$ minutes. Fluoroscopy time was extremely short, at 5.4 \pm 3.7 minutes. ${ }^{37}$ Even if procedure times were longer in cases of persistent AFib (+10\%), fluoroscopy times remained unchanged (5.3 \pm 3.5 versus $5.6 \pm 4$ minutes; $P=0.7) .{ }^{37}$ The rate of serious complications was very low $(0.7 \%)$.

Limitations have been raised about the technique's usefulness in the ablation of flutter, in which procedure times 
are significantly longer compared with the conventional method. ${ }^{35}$ Prospective data on treatment using the robotic technique are beginning to be validated in the treatment of ventricular tachycardia. ${ }^{38-40} \mathrm{~A}$ recent study has compared the manual $(n=80)$ and robotic methods and has shown the robotic method to be superior in the treatment of ischemic ventricular tachycardia over a follow-up period of 15 months, with a recurrence rate of $41 \%$ versus $19 \%$, respectively. ${ }^{37}$ While the results appear to be similar in other indications, such as dilated cardiomyopathy or dysplasia, the utility of RMNS in the epicardial approach is widely reported in the international literature. ${ }^{38,39}$ Skoda et al. also studied a prospective cohort of 53 patients with ischemic ventricular tachycardia. ${ }^{40}$ These authors showed an efficacy rate of $94.2 \%$, a recurrence rate of $38 \%$, and a procedure time of only 8.65 minutes with no complications. ${ }^{40}$

\section{Advantages of the robotic technique \\ Fluoroscopy}

One of the main advantages that emerges from our literature review is the very significantly reduced X-ray exposure time when using the RMNS system..$^{9-16,35-40}$ In the short term, this may appear to patients to be of little relevance, but in the event of multiple interventions accompanied by irradiating examinations, this may represent a very significant reduction in radiation. ${ }^{35}$ This finding is even truer for electrophysiologists, whose work is sometimes multidisciplinary, and include implanting resynchronization devices and ablating complex arrhythmias. The long-term benefits are all too clear. ${ }^{35}$ The extent of the reduction in X-ray exposure has been estimated to be, on average, $50 \% .{ }^{35}$ Very similar results were published by Kim et $\mathrm{al}^{41}$, showing a mean reduction of 29 minutes compared with the conventional method. This represents an advantage for both the patient, in the event of a complex procedure or a repeated procedure, and the operator, for whom chronic exposure poses a significant risk. ${ }^{19}$

\section{Procedure time}

Procedure times seem to be the same with RMNS and the conventional methods, but a certain number of biases exist in the published studies. These studies were conducted with the most experienced teams in Europe and the world. Several operators were generally involved, some of whom were fellows, and the learning curve was taken into account, which makes analyzing the results difficult. While procedure time seems to be a deciding factor, operator fatigue should probably be included in the equation. All things considered, we believe that RMNS has been of major benefit in this regard, enabling us to increase our work very considerably while reducing fatigue at the end of the day.

\section{Safety}

Using the RMNS magnetic catheter, whose flexibility is of a major advantage, has considerably increased the safety of complex procedures such as AFib RFA..$^{9-16,35}$ The risk of perforation is practically zero owing to the constant force exerted on the tissue, which has been evaluated to be a maximum of 15-20 g. ${ }^{9-16,35}$ Catheter-related cases of tamponade represent the exceptions in the literature. ${ }^{35}$ Using this tool in daily practice for all our AFib ablation procedures has given our center the greatest peace of mind. Approximately, more than 1,000 cases of AFib ablation were conducted in our department with the stereotaxis system, and we have observed no cases of tamponade following RMNS. One of the theoretical advantages that is, in our opinion, currently unquantifiable is that the sheath is not repeatedly manipulated. Manipulation of the sheaths is greatly reduced by the robotic technique, and occurs only when the lasso is moved from one vein to another. This may limit the risk of thrombosis or embolism. If we compare the two robotic systems, they differ significantly in terms of their technology: the magnetic catheter is nontraumatic whereas the catheter used with the Hansen system remains a conventional catheter with contact force measurement. In this regard, the tamponade rate appears to be higher with the second system.

\section{Efficacy}

The results presented in the various studies appear to be similar to the conventional method, but they are not as yet, as initially expected, superior. ${ }^{35-39}$ They should be interpreted in light of current circumstances, in which operators are far less experienced with this new approach than they are with the manual method. ${ }^{35}$ We must wait for prospective studies conducted in experienced centers using the two methods to properly answer this question. Certain theoretical advantages appear to favor RMNS, such as the stability of the catheter, precision, access to difficult areas like the right inferior pulmonary vein, the quality of the lines made, and the homogeneity of the lesions. ${ }^{35}$ Magnetic navigation's utility has made it the treatment of choice for cardiac rhythm disorders in congenital heart disease. ${ }^{42}$

\section{Cost and comfort of use}

Overall comfort of use is excellent. One main advantage lies in joystick manipulation: the operator directs the catheter by means of a vector on the 3D mapping screen, and then the magnetic field moves the catheter in the direction indicated 
by the operator. This indirect manipulation is precise and as quick as direct manipulation. Current technical developments of RMNS have made it possible to market a new platform, EPOCH, which allows "almost" instantaneous movement of the magnetic catheter. This considerably reduces procedure times. The option of having automated tools with the magnetic robot has also resulted in saving time. For instance with the Naviline system, automated lines can be made for RFA procedures of AFib, and particularly persistent AFib; or there is the automated processing of a vein with "go to the electrode", which enables the catheter to be steered to the dipole selected in one click. This is an ideal tool for segmentally isolating veins. Other high-performance tools may be used with this magnetic robot system and are undergoing constant development. This should further improve ablation results and also reduce procedure times.

\section{Limitations of stereotaxis}

The main drawback of RMNS lies in the cost of installation, which may amount to up to $€ 1.5$ million if we take into account the construction of a dedicated room. This cost should be weighed against the many long-term advantages such as efficacy, safety and, once again, the reduction of X-ray exposure. This futuristic technology adapts perfectly to the treatment of complex arrhythmias, particularly AFib RFA. The second drawback is the "closed" nature of the system, which is only compatible with Cordis Webster catheters and mapping systems such as CARTO XP, CARTOMERGE, and CARTO 3 RMT.

The presence of a cardiac stimulation or defibrillation device makes the operator reticent to perform a stereotaxis procedure ${ }^{43,44} \mathrm{~A}$ recent study has shown that the risk of interference was in fact very low, since $95 \%$ of the 121 devices tested showed no interference with parameter settings, battery status, or data recorded..$^{42}$ Interference was observed in six patients with pacemakers, resulting in reprogramming only. ${ }^{43}$ Eitel et al published a retrospective study involving 31 patients with pacemakers $(n=5)$ and defibrillators $(n=26)$ showing the system's safety in these patients, with the usual caveats pertaining to the small patient number in the study. ${ }^{44}$ These results corroborate findings that the magnetic field exerted by the stereotaxis system is $20-40$ times weaker than in magnetic resonance imaging. ${ }^{44}$

Other limitations were raised by Miyazaki et a ${ }^{15}$ for the AFib treatment, such as a longer set-up time, the need for the operator to move when positioning his lasso, the low contact force, which can limit the size of the lesion, and the use of an additional sheath for ablation, as well as the cost of the procedure compared with the manual lasso technique. These disadvantages are not seen in the highly experienced teams, especially since significant developments have been made over recent months with the release of the latest version of the Niobe ES system (EPOCH), which has increased magnetic catheter execution speed by $500 \%$ compared with the previous version, as well as the marketing of the V-drive system, which enables automated remote lasso manipulation. What is more, a recent study compared RMNS ablation and contact force in AFib ablation. The results between manual ablation $(n=312)$ and RMNS ablation $(n=315)$ were similar, with the respective recurrence rates being $36.8 \%$ versus $38.6 \%(P=1)$, but radiation exposure rates were lower with RMNS. ${ }^{45}$

Finally some users feel they do not get sufficient sustained contact pressure with stereotaxis catheters with endocardium and that might increase likelihood of gaps in ablation lines. On the other side, experimental and clinical studies argue against this theory with similar results compared with manual ablation.

\section{Conclusion}

The treatment of complex arrhythmias such as AFib will account for most ablations in the future in electrophysiology departments. Over recent years, robotic magnetic navigation has emerged as a modern technology that is particularly suited to the treatment of these complex arrhythmias given that its efficacy is comparable to the manual technique in all clinical trials and it considerably reduces complications and X-ray exposure. The magnetic robotic technique offers fairly clear advantages for both patients and operators, whose constraints are very significantly reduced when using these systems.

\section{Disclosure}

The authors report no conflicts of interest in this work.

\section{References}

1. Haissaguerre M, Jais P, Shah DC, et al. Spontaneous initiation of atrial fibrillation by ectopic beats originating in the pulmonary veins. $N \mathrm{Engl}$ J Med. 1998;339(10):659-666.

2. Terasawa T, Balk EM, Chung M, et al. Systematic review: comparative effectiveness of radiofrequency catheter ablation for atrial fibrillation. Ann Intern Med. 2009;151(3):191-202.

3. Reynolds MR, Zimetbaum P, Josephson ME, Ellis E, Danilov T, Cohen DJ. Cost-effectiveness of radiofrequency catheter ablation compared with antiarrhythmic drug therapy for paroxysmal atrial fibrillation. Circ Arrhythm Electrophysiol. 2009;2(4):362-369.

4. Wyse DG. Rhythm versus rate control trials in atrial fibrillation. J Cardiovasc Electrophysiol. 2003;14(Supp1 9):S35-S39.

5. Corely SD, Epstein AE, DiMarco JP, et al. Relationships between sinus rhythm, treatment and survival in the Atrial Fibrillation Follow-up Investigation of Rhythm Management (AFFIRM) Study. Circulation. 2004;109(12):1509-1513.

6. Brand FN, Abbott RD, Kannel WB, Wolf PA. Characteristics and prognosis of lone atrial fibrillation: 30 -year follow-up in the Framingham Study. JAMA. 1985;254(24):3449-3453. 
7. Wyse DG, Waldo AL, DiMarco JP, et al. A comparison of rate control and rhythm control in patients with atrial fibrillation. $N$ Engl J Med. 2002; 347(23):1825-1833.

8. Steinberg JS, Sadaniantz A, Kron J, et al. Analysis of cause-specific mortality in the Atrial Fibrillation Follow-up Investigation of Rhythm Management (AFFIRM) Trial. Circulation. 2004;109(16): 1973-1980.

9. Pappone C, Vicedomini G, Manguso F, et al. Robotic magnetic navigation for atrial fibrillation ablation. $J$ Am Coll Cardiol. 2006;47(7): $1390-1400$

10. Di Biase L, Fahmy TS, Patel D, et al. Remote magnetic navigation: human experience in pulmonary vein ablation. $J$ Am Coll Cardiol. 2007;50(9):868-874.

11. Schmidt B, Chun KR, Tilz RR, Koektuerk B, Ouyang F, Kuck $\mathrm{KH}$. Remote navigation systems in electrophysiology. Europace. 2008;10(Suppl 3):57-61.

12. Katsiyiannis WT, Melby DP, Matelski JL, Ervin VL, Laverence KL, Gornick CC . Feasbility and safety of remote-controlled magnetic navigation for ablation of atrial fibrillation. Am J Cardiol. 2008;102(12):1674-1676.

13. Chun KR, Wissner E, Koektuerk B, et al. Remote-controlled magnetic pulmonary vein isolation using a new irrigated-tip catheter in patients with atrial fibrillation. Circ Arrhythm Electrophysiol. 2010;3(5):458-464.

14. Luthje L, Vollmann D, Seegers J, et al. Remote magnetic versus manual catheter navigation for circumferential pulmonary vein ablation in patients with atrial fibrillation. Clin Res Cardiol. 2011;100(11):1003-1011.

15. Miyazaki S, Shah AJ, Xhaët O, et al. Remote navigation with irrigated tip catheter for ablation of paroxysmal atrial fibrillation. Circ Arrhythm Electrophysiol. 2010;3(6):585-588.

16. Paponne C, Vicedomini G, Frigoli E, et al. Irrigated-tip magnetic catheter ablation of AF: a long-term prospective study in 130 patients. Heart Rhythm. 2011;8(1):8-15.

17. Hlivák P, Mlčochová H, Peichl P, Cihák R, Wichterle D, Kautzner J. Robotic navigation in catheter ablation for paroxysmal atrial fibrillation: midterm efficacy and predictors of postablation arrhythmia recurrences. J Cardiovasc Electrophysiol. 2011;22(5):534-540.

18. Malcolme-Lawes L, Kanagaratnam P. Robotic navigation and ablation. Minerva Cardioangiol. 2010;58(6):691-699.

19. Steven D, Servatius H, Rostock T, et al. Reduced fluoroscopy during atrial fibrillation ablation: benefits of robotic guided navigation. $J$ Cardiovasc Electrophysiol. 2010;21(1):6-12.

20. Rillig A, Schmidt B, Steven D, et al. Study design of the man and machine trial: a prospective international controlled noninferiority trial comparing manual with robotic catheter ablation for treatment of atrial fibrillation. J Cardiovasc Electrophysiol. 2013;24:40-6.

21. Bai R, DI Biase L, Valderrabano M, et al. Worldwide experience with the robotic navigation system in catheter ablation of atrial fibrillation: methodology, efficacy and safety. $J$ Cardiovasc Electrophysiol. 2012;23(8):820-826.

22. Koa-Wing M, Kojodjojo P, Malcolme-Lawes LC, et al. Robotically assisted ablation produces more rapid and greater signal attenuation than manual ablation. J Cardiovasc Electrophysiol. 2009;20(12): $1398-1404$.

23. Steven D, Servatius H, Rostock T, et al. Reduced fluoroscopy during atrial fibrillation ablation: benefits of robotic guided navigation. J Cardiovasc Electrophysiol. 2010;21(1):6-12.

24. Wazni OM, Barrett C, Martin DO, et al. Experience with the Hansen robotic system for atrial fibrillation ablation - lessons learned and techniques modified: Hansen in the real world. J Cardiovasc Electrophysiol. 2009;20(11):1193-1196.

25. Di Biase L, Natale A, Barrett C, et al. Relationship between catheter forces, lesion characteristics, "popping," and char formation: experience with robotic navigation system. $J$ Cardiovasc Electrophysiol. 2009;20(4):436-440.
26. Saliba W, Reddy VY, Wazni O, et al. Atrial fibrillation ablation using a robotic catheter remote control system: initial human experience and long-term follow-up results. J Am Coll Cardiol. 2008;51(25):2407-2411.

27. Tillander $\mathrm{H}$. Magnetic guidance of a catheter with articulated steel tip. Acta Radiol. 1951;35(1):62-64.

28. Ram W, Meyer H. Heart catheterization in a neonate by interacting magnetic fields: a new and simple method of catheter guidance. Cathet Cardiovasc Diagn. 1991;22(4):317-319.

29. Gillies GT, Ritter RC, Broaddus WC, Grady MS, Howard MA, McNeil RG. Magnetic manipulation instrumentation for medical physics research. Rev Sci Instrum. 1994;65:533-562.

30. Faddis MN, Blume W, Finney J, et al. Novel magnetically guided catheter for endocardial mapping and radiofrequency catheter ablation. Circulation. 2002;106:2980-2985.

31. Faddis MN, Chen J, Osborn J, Talcott M, Cain ME, Lindsay BD. Magnetic guidance system for cardiac electrophysiology: a prospective trial of safety and efficacy in humans. $J$ Am Coll Cardiol. 2003;42(11):1952-1958.

32. Saliba W, Cummings JE, Oh S, et al. Novel robotic catheter remote control system: feasibility and safety of transseptal puncture and endocardial catheter navigation. J Cardiovasc Electrophysiol. 2006;17(10): 1102-1105.

33. Okumura Y, Johnson SB, Bunch TJ, Henz BD, O'Brien CJ, Packer DL. A systematical analysis of in vivo contact forces on virtual catheter tip/tissue surface contact during cardiac mapping and intervention. J Cardiovasc Electrophysiol. 2008;19(6):632-640.

34. Cappato R, Calkins H, Chen SA et al. Updated worldwide survey on the methods, efficacy and safety of catheter ablation for human atrial fibrillation. Circ Arrhythm Electrophysiol. 2010;3(1):32-38.

35. Bradfield J, Tung R, Mandapati R, Boyle NG, Shivkumar K. Catheter ablation utilizing remote magnetic navigation: a review of applications and outcomes. Pacing Clin Electrophysiol. 2012;35(8):1021-1034.

36. Adragao PP, Cavaco D, Ferreira AM, et al. Safety and long-term outcomes of catheter ablation of atrial fibrillation using magnetic navigation versus manual conventional ablation: a propensity-score analysis. $J$ Cardiovasc Electrophysiol. 2016;27(Suppl 1):S11-S16.

37. Jin QI, Pehrson S, Jacobsen PK, Chen XU. Efficacy and safety of atrial fibrillation ablation using remote magnetic navigation: experience from 1006 procedures. J Cardiovasc Electrophysiol. 2016;27(Suppl 1):S23-S28.

38. Di Biase L, Burkhardt DJ, Mohanty P, et al. Scar homogeneization ablation in patients with ischemic cardiomyopathy: comparison between remote magnetic ablation and manual ablation. Circulation. 2015; 132:A14384.

39. Aagaard P, Natale A, Briceno D, et al. Remote magnetic navigation: a focus on catheter ablation of ventricular arrhythmias. J Cardiovasc Electrophysiol. 2016;27(Suppl 1):S38-S44.

40. Skoda J, Arya A, Garcia F, et al. Catheter ablation of ischemic ventricular tachycardia with remote magnetic navigation: STOP-VT multicenter trial. J Cardiovasc Electrophysiol. 2016;27(Suppl 1):S29-S37.

41. Kim AM, Turakhia M, Lu J, et al. Impact of remote magnetic navigation on ablation fluoroscopic and procedure time. Pacing Clin Electrophysiol. 2008;31(11):1399-1404.

42. Roy K, Gomez-Pulido F, Ernst S. Remote magnetic navigation for catheter ablation in patients with congenital heart disease: a review. J Cardiovasc Electrophysiol. 2016;27(Suppl 1):S45-S56.

43. Jilek C, Tzeis S, Reents T, et al. Safety of implantable pacemakers and cardioverter defibrillators in the magnetic field of a novel remote magnetic navigation system. J Cardiovasc Electrophysiol. 2010;21(10): $1136-1141$.

44. Eitel C, Hindricks G, Sommer P, et al. Safety of remote magnetic navigation in patients with pacemakers and implanted cardioverter defibrillators. J Cardiovasc Electrophysiol. 2010;21(10):1130-1135.

45. Weiss JP, May HT, Bair TL, et al. A comparison of remote magnetic irrigated tip ablation versus manual catheter irrigated tip catheter ablation with and without force sensing feedback. J Cardiovasc Electrophysiol. 2016;27(Suppl 1):S5-S10. 
Medical Devices: Evidence and Research is an international, peerreviewed, open access journal that focuses on the evidence, technology, research, and expert opinion supporting the use and application of medical devices in the diagnosis, monitoring, treatment and management of clinical conditions and physiological processes. The identification of novel devices and optimal use of existing devices which will lead to improved clinical outcomes and more effective patient management and safety is a key feature. The manuscript management system is completely online and includes a quick and fair peer-review system. Visit http://www. dovepress.com/testimonials.php to read real quotes from authors.

Submit your manuscript here: https://www.dovepress.com/medical-devices-evidence-and-research-journal 